\title{
Association between new-onset liver cirrhosis and suicide risk in South Korea: A nationwide cohort study
}

Suk-Yong Jang ${ }^{1, *}$, Woo Sun Rou ${ }^{2,3, \dagger}$, Seok Hyun Kim ${ }^{2,3}$, Byung Seok Lee ${ }^{2,3}$, and Hyuk Soo Eun ${ }^{2,3}$

${ }^{1}$ Department of Preventive Medicine, Eulji University College of Medicine, Daejeon; ${ }^{2}$ Department of Internal Medicine, Chungnam National University School of Medicine, Daejeon; ${ }^{3}$ Department of Internal Medicine, Chungnam National University Hospital, Daejeon, Korea

\section{Graphical Abstract}

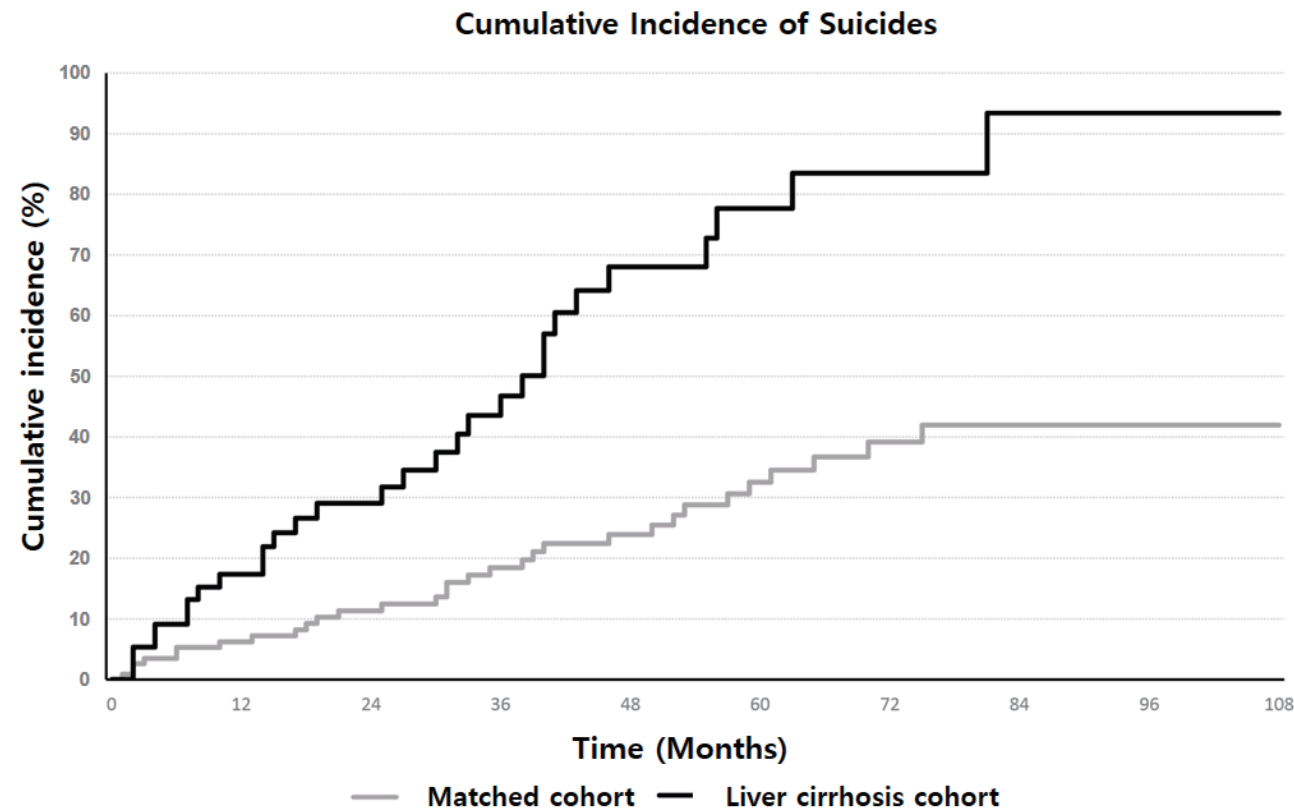

\section{Abbreviations:}

CCl, Charlson Comorbidity Index; Cl, confidence interval; HR, hazard ratio; ICD-10, International Statistical Classification of Diseases and Related Health Problems, 10th Revision; IR, incidence rate; LC, liver cirrhosis; NHID, National Health Information Database; NHIS-NSC, National Health Insurance Service-National Sample Cohort; OR, odds ratio; SMR, standardized mortality ratios

* Current affiliation: Department of Healthcare Management, Graduate School of Public Health, Yonsei University, Seoul, Korea

${ }^{\dagger}$ Current affiliation: Department of Internal Medicine, Chungnam National University Sejong Hospital, Sejong, Korea

Editor: Salvatore Piano, University of Padova Faculty of Medicine and Surgery, Italy
Corresponding author: Hyuk Soo Eun

Department of Internal Medicine, Chungnam National University School of Medicine, 266 Munhwa-ro, Jung-gu, Daejeon 35015, Korea

Tel: +82-42-280-7418, Fax: +82-42-222-7141

E-mail:hyuksoo@cnuh.co.kror hyuksoo@cnu.ac.kr https://orcid.org/0000-0003-0485-0072

Received: Aug. 14, 2020 / Revised : Nov. 19, 2020 / Accepted : Nov. 23, 2020

Copyright ( 2021 by Korean Association for the Study of the Liver

This is an Open Access article distributed under the terms of the Creative Commons Attribution Non-Commercial License (http://creativecommons.org/licenses/by-nc/3.0/) which permits unrestricted non-commercial use, distribution, and reproduction in any medium, provided the original work is properly cited. 
Background/Aims: Current evidence suggests that liver cirrhosis (LC) causes severe psychological stress and depression, which are risk factors for suicide. Although previous studies reported the association between $\mathrm{LC}$ and suicidal thoughts, little is known of its effect on suicidal deaths. Therefore, this study was undertaken to investigate the effect of new-onset LC on suicide.

Methods: From the National Health Insurance Service-National Sample Cohort of South Korea, 5,809 incident LC patients and 11,618 risk-set controls matched by propensity score were selected for follow-up. The incidence rate of suicide was estimated using a generalized estimating equation with a Poisson distribution. Effect size was presented as a hazard ratio (HR) using Cox's proportional hazards model.

Results: The incidence rate of suicide was 143.3 cases per 100,000 person years ( $95 \%$ confidence interval [Cl], 100.2205.1) among the LC cohort. The LC patients were 2.37 times more likely to commit suicide compared with matched controls ( $\mathrm{HR}, 2.37 ; 95 \% \mathrm{Cl}, 1.44-3.88)$. Increased suicide risk was evident within the first 2 years of the follow-up period (HR, $2.59 ; 95 \% \mathrm{Cl}, 1.20-5.60)$ and among the $18-49$-year-old age group ( $\mathrm{HR}, 3.72 ; 95 \% \mathrm{Cl}, 1.45-9.56)$.

Conclusions: Our study found increased risk of suicide in patients with new onset LC, especially during the early period following diagnosis and in younger patients. To decrease this suicide risk, a regular and continuous social support system is required. (Clin Mol Hepatol 2021;27:283-294)

Keywords: Liver cirrhosis; Suicide; Risk; Depression; Psychological distress

\footnotetext{
Study Highlights

- Patients newly diagnosed with cirrhosis face severe psychological stress.

- Patients newly diagnosed with cirrhosis have a 2.37 times higher suicidal incidence rate than the matched cohort group.

- A social and psychological support system is required for patients diagnosed with cirrhosis.
}

\section{INTRODUCTION}

Chronic illnesses are strongly correlated with suicide risk. 'The risk of suicide in people suffering from diseases, such as cancer, chronic renal failure, or heart failure, is significantly higher than that of the general population. ${ }^{2-4}$ However, few reports have investigated the risk of suicide resulting from chronic liver diseases, such as liver cirrhosis (LC).

Diagnosis of LC can cause significant psychological stress in patients. This is, in part, because most forms of $L C$ are irreversible, and the disease brings about a significant decrease in quality of life, which is consequent to complications arising from deterioration of hepatic function. Accumulation of psychological distress may not only cause depression, but also lead to suicidal thoughts. Indeed, a previous study has reported that patients with chronic liver disease have a higher risk of depression and demonstrate increased attempts at suicide. ${ }^{5}$

The most common cause of $\mathrm{LC}$ in South Korea is chronic viral hepatitis B, followed by alcoholic hepatitis and chronic viral hepatitis C. ${ }^{6}$ The prevalence of chronic hepatitis B-related LC has continued to decrease due to vaccination and administration of anti- viral drugs. However, the prevalence of alcoholic LC has continued to increase to $31 \%$, and this prevalence is high compared with that of other developed countries. ${ }^{6}$ In 2015, socioeconomic costs to the 40-49-year-old age group were the second highest burden of $L C$ after those for self-harm, and it is suggested that the socioeconomic burden associated with LC is very high. ${ }^{\text {H However, few }}$ organizations or committees offer psychological health support to patients with LC.

When comparing the absolute risk of suicide to death resulting from LC complications, the risk may be significantly lower. However, although LC symptoms cannot always be effectively controlled, psychological intervention can be provided to prevent suicides. Indeed, several studies suggest that psychological stress in patients with LC is clinically relevant. Specifically, patients with LC have psychological distress and depression, which are correlated with severity of liver disease. ${ }^{8-11}$ Moreover, several studies have suggested that severe psychological stress, such as depression, impairs the clinical prognosis of patients with chronic liver diseases and LC, as well as that of post-liver transplant patients. ${ }^{12-14}$ However, few studies have investigated whether the risk of suicide is higher in LC patients. Our study attempts to fill this gap by 
investigating whether newly developed LC increases the risk of suicide.

\section{MATERIALS AND METHODS}

\section{Study subjects}

Incident LC patients and matched controls were selected from the National Health Insurance Service-National Sample Cohort (NHIS-NSC) of South Korea. The NHIS established an administrative database for research purposes, i.e., the National Health Information Database (NHID), which stores all healthcare service records from the general population. ${ }^{15,16}$ From the NHID, the NHIS constructs and provides researchers the NHIS-NSC, a representative administrative dataset, for guiding health policy and carrying out biomedical research. A detailed cohort profile was published by the NHIS. ${ }^{16}$ The NHIS-NSC consists of one million people selected by a systematic stratified random sampling method from a target population of $48,222,537$ in 2006 . Under the aegis of a compulsory social insurance system instituted by the National Health Insurance Act, all subjects could be followed until 2015, except in cases of disqualification due to death or emigration. Data from 2002 to 2005 were included to provide additional past medical information. As a single payer, the NHIS maintains all personal information, demographics, and medical treatment data for the entire Korean population. Key variables in the NHIS-Senior include all inpatient and outpatient medical claims data, such as treatment procedure codes, prescription codes, and diagnostic codes. This study protocol was approved by the Institutional Review Board of Chungnam National University Hospital (IRB No.: 2019-10-001).

\section{Incident LC cohort}

The incident LC cohort was constructed from the NHIS-NSC base cohort. To be enrolled, subjects must fit the operational definition of new-onset $L C$, i.e., first-time admission to an acute care hospital or two or more outpatient visits with primary or the first secondary diagnostic codes of LC (International Statistical Classification of Diseases and Related Health Problems, 10th Revision [ICD-10] K702, K703, K717, K74, K761, K765, K766). The decompensated LC cohort comprised those with K702, K703, K717, K74, K761, K765, K766 codes accompanying 185.0, 186.4, 198.3, K72, K72.1, K72.9, K76.7, R17, R18 codes, and the alcoholic cirrhosis cohort was defined as patients with simultaneous codes of K70.2 and K70.3. For definition of the ICD-10 code for LC patient groups, validation studies and a South Korean study were referenced. ${ }^{17-20}$ Among these patients, some were excluded to maintain the study design validity. To select new-onset LC cases, patients having medical claims of LC between 2002 and 2006 (a 5-year washout period) were considered to have a diagnosis of LC previously, and were excluded. The date of LC incidence was defined as that of the first admission or the second outpatient visit, whichever occurred first. The second outpatient visit was considered the date of diagnosis of LC to avoid immortal time bias, ${ }^{21}$ which is the interval between the first and the second outpatient visit. LC patients younger than 18 years at the time of LC incidence were also excluded. From January 1, 2007, new-onset LC patients were enrolled in the incident $\mathrm{LC}$ cohort at the index date (time zero) and followed up.

\section{Identification of suicides}

Information on death from Statistics Korea was individually linked using unique personal identification numbers. ${ }^{16,22}$ Under a de-identification policy, the calendar year and month of death were provided by the NHIS, after masking the exact date. By law all deaths are required to be reported to Statistics Korea. Suicide was identified by the set of deaths assigned 'Intentional selfharm' (ICD-10 codes, X60 to X84).

\section{Risk set matching on propensity score}

Although the NHIS-NSC was constructed retrospectively, the design of this study was planned to mimic a prospective study. First, a time-dependent propensity score was calculated, followed by risk-set matching. ${ }^{23,24}$ To adjust for confounding effects, the association between LC and suicide was examined by time-dependent propensity score matching. ${ }^{24}$ Hazard components (as propensity score) were estimated using Cox's proportional hazards model with January 1, 2007 as baseline and LC as event. All variables included in Table 1 were independent variables and were collected from 3 years (2004 to 2006) before baseline. Age and square of age were included as continuous variables, whereas sex, household income level (decile), registered disability, residential district as fixed effect, Charlson Comorbidity Index (CCI), numbers of hospital admissions and outpatient visits, and past medical history were included as categorical variables. Each subject's number of comorbidities was assessed by diagnostic codes using the Quan 


\section{CLINCAL and MOLECULAR}

Volume_27 Number_2 April 2021

Table 1. Baseline characteristics of liver cirrhosis patients and their risk set-matched cohort

\begin{tabular}{|c|c|c|c|}
\hline Characteristic & Liver cirrhosis cohort $(n=5,552)$ & Matched cohort $(n=11,104)$ & Standardized difference \\
\hline Age (years) & $56.3 \pm 12.9$ & $56.3 \pm 12.9$ & 0 \\
\hline Square of age & $3,334.2 \pm 1,472.8$ & $3,334.2 \pm 1,472.8$ & 0 \\
\hline Female sex & $1,586(28.6)$ & $3,172(28.6)$ & 0 \\
\hline Household income level (decile) & & & 0.0314 \\
\hline Medical aid program & $447(8.1)$ & $942(8.5)$ & \\
\hline \multicolumn{4}{|l|}{ National health insurance } \\
\hline First & $478(8.6)$ & $976(8.8)$ & \\
\hline Second & $450(8.1)$ & $966(8.7)$ & \\
\hline Third & $429(7.7)$ & $855(7.7)$ & \\
\hline Fourth & $505(9.1)$ & $982(8.8)$ & \\
\hline Fifth & $476(8.6)$ & $944(8.5)$ & \\
\hline Sixth & $449(8.1)$ & $887(8.0)$ & \\
\hline Seventh & $644(11.6)$ & $1,260(11.3)$ & \\
\hline Eighth & $475(8.6)$ & $937(8.4)$ & \\
\hline Ninth & $583(10.5)$ & $1,124(10.1)$ & \\
\hline Tenth & $616(11.1)$ & $1,231(11.1)$ & \\
\hline Registered disability & $474(8.5)$ & $923(8.3)$ & 0.0081 \\
\hline Residential district & & & 0.0307 \\
\hline Seoul & $1,000(18.0)$ & $1,971(17.8)$ & \\
\hline Pusan & $503(9.1)$ & $982(8.8)$ & \\
\hline Daegu & $241(4.3)$ & $501(4.5)$ & \\
\hline Incheon & $247(4.4)$ & $496(4.5)$ & \\
\hline Gwangju & $159(2.9)$ & $307(2.8)$ & \\
\hline Daejeon & $120(2.2)$ & $225(2.0)$ & \\
\hline Ulsan & $119(2.1)$ & $231(2.1)$ & \\
\hline Gyeonggi-do & $1,102(19.9)$ & $2,209(19.9)$ & \\
\hline Gangwon-do & $210(3.8)$ & $432(3.9)$ & \\
\hline Chungcheongbuk-do & $145(2.6)$ & $296(2.7)$ & \\
\hline Chungcheongnam-do & $279(5.0)$ & $525(4.7)$ & \\
\hline Jeollabuk-do & $259(4.7)$ & $522(4.7)$ & \\
\hline Jeollanam-do & $340(6.1)$ & $732(6.6)$ & \\
\hline Gyeongsangbuk-do & $331(6.0)$ & $656(5.9)$ & \\
\hline Gyeongsangnam-do & $417(7.5)$ & $859(7.7)$ & \\
\hline Jeju-do & $80(1.4)$ & $160(1.4)$ & \\
\hline Charlson Comorbidity Index score & & & 0.0450 \\
\hline 0 & $3,082(55.5)$ & $6,300(56.7)$ & \\
\hline 1 & $1,345(24.2)$ & $2,745(24.7)$ & \\
\hline 2 & $624(11.2)$ & $1,162(10.5)$ & \\
\hline 3 & $291(5.2)$ & $509(4.6)$ & \\
\hline 4 or more & $210(3.8)$ & 388 (3.5) & \\
\hline
\end{tabular}


Table 1. Continued

\begin{tabular}{|c|c|c|c|}
\hline Characteristic & Liver cirrhosis cohort $(n=5,552)$ & Matched cohort $(n=11,104)$ & Standardized difference \\
\hline Number of hospital admission & & & 0.0412 \\
\hline 0 & $4,047(72.9)$ & $8,276(74.5)$ & \\
\hline 1 & $876(15.8)$ & $1,694(15.3)$ & \\
\hline 2 or more & $629(11.3)$ & $1,134(10.2)$ & \\
\hline Number of outpatient visit & & & 0.0616 \\
\hline 0 & $329(5.9)$ & $720(6.5)$ & \\
\hline $1-9$ & $1,590(28.6)$ & $3,383(30.5)$ & \\
\hline $10-19$ & $1,153(20.8)$ & $2,360(21.3)$ & \\
\hline $20-29$ & $747(13.5)$ & $1,384(12.5)$ & \\
\hline $30-39$ & $578(10.4)$ & $1,056(9.5)$ & \\
\hline 40 or more & $1,155(20.8)$ & 2,201 (19.8) & \\
\hline Antihypertensive agents & $1,392(25.1)$ & $2,609(23.5)$ & 0.0368 \\
\hline Antidiabetic agents & $598(10.8)$ & $1,085(9.8)$ & 0.0329 \\
\hline Lipid lowering agents & $444(8.0)$ & $865(7.8)$ & 0.0077 \\
\hline Antidepressive agents & $400(7.2)$ & $800(7.2)$ & 0 \\
\hline Antiviral agents & $138(2.5)$ & $267(2.4)$ & 0.0052 \\
\hline Malignant neoplasm & $185(3.3)$ & $326(2.9)$ & 0.0227 \\
\hline Congestive heart failure & $67(1.2)$ & $112(1.0)$ & 0.0189 \\
\hline Chronic obstructive pulmonary disease & $472(8.5)$ & $887(8.0)$ & 0.0187 \\
\hline Alcoholic liver disease & $385(6.9)$ & $798(7.2)$ & 0.0098 \\
\hline
\end{tabular}

Values are presented as mean \pm standard deviation or number $(\%)$.

At the date of liver cirrhosis incidence of each patient, two controls were matched on follow-up time and propensity score estimated by Cox proportional hazards model with predictors included in this table.

ICD-10 coding algorithm of $\mathrm{CCl}$ score. ${ }^{25}$ The presence of $\mathrm{CCl}$ disease-constituting categories and the presence of a past medical history of malignant neoplasm, congestive heart failure, chronic obstructive pulmonary disease, or alcoholic liver disease were defined by at least two outpatient visits or one admission upon primary or first secondary diagnosis. Prescription over 90 days for antihypertensive, antidiabetic, or lipid-lowering agents was accounted for as patients using corresponding medications.

Second, to emulate a prospective study, the patients were matched to subjects at risk of LC by age, sex, and use of anti-depressants at the calendar date (time zero) when the first LC patient was identified. This method of risk-set matching was repeated for subsequent patients until the last LC patient. 23,26,27

Third, 1:2 matching on propensity score was performed sequentially for each risk set using a nearest neighbor matching algorithm with a maximum caliber of 0.1 of the hazard components. To allow matching to be independent of future events, the matched-control subjects could be either those who never developed LC or those who had not yet developed LC. Thus, an LC patient in the incident $\mathrm{LC}$ cohort could enter the study as an LC patient or a matched control for another LC patient whose time zero was prior to that of the initial patient. ${ }^{28}$ Next, to yield non-overlapping samples from the risk set, the matched subjects were removed from the next risk sets. The process was performed iteratively with the next risk sets until there were no more treated patients in the risk set.

\section{Statistical analyses}

Using the final matched cohorts, statistical tests for the association between incident LC and risk of suicide were performed considering the statistical nature of matched-pair analysis. To assess covariate balance between treatment groups, baseline characteristics were compared with standardized differences where a 


\section{CLINICAL and MOLECULAR}

\section{HEPATOLOGY}

difference less than $0.1(10 \%)$ is generally considered negligible. ${ }^{29,30}$ The cumulative incidence curve of suicide was obtained by Kaplan-Meier survival curves and a stratified log-rank test was used to compare the Kaplan-Meier curves of the matched cohort. ${ }^{31}$ Cumulative incidence of suicide and 95\% confidence interval $(\mathrm{Cl})$ was calculated by the product limit (Kaplan-Meier) method of survival probability. Incidence rate (IR) of suicide and 95\% Cl were calculated based on a generalized estimating equation with a Poisson distribution and expressed as number of suicides per 100,000 person-years. The effect size was presented as hazard ratio (HR) using Cox's proportional hazards model with a robust variance estimator that accounts for clustering within matched pairs. ${ }^{30,31}$ Time zero was set to the date of $\mathrm{LC}$ incidence for both LC patients and their matched controls. Survival time used in analyses was defined as months from time zero to time of death or December 31, 2015, whichever came first. Proportional hazards assumption was assessed by graphically using log transformation of the negative log of estimated survivor function, time-dependent explanatory variables, Schoenfeld residuals, cumulative sums of martingale residuals, and a supremum test. In addition to these cause-specific models for competing risks, a Fine and Gray sub-distribution hazard model was performed as a sensitivity analysis. Sub-group analyses within categorized age groups (18-49, 50-64, and 65 years or more), sex, and followup time frames $(0-24,25-60,61$ months or over) were per- formed. To compare the suicide rate according to severity of cirrhosis, the analysis was performed by dividing patients into matched cohort, compensated LC, and decompensated LC. Moreover, to additionally perform stratified analysis on patients with depression, as a coexisting mental illness, the suicide rate according to presence or absence of LC was analyzed by categorizing whether anti-depressant was administered. The statistical analyses were conducted using SAS Enterprise Guide version 7.1 (SAS Institute, Cary, NC, USA). $P$-values $<0.05$ were considered statistically significant.

\section{RESULTS}

\section{Flow of analysis and baseline characteristics of patients}

From January 1, 2002 to December 31, 2015, a total of 10,594 patients met the inclusion criteria. Among these patients, LC patients during the washout period of 2002 to 2006 (4,354 patients) were excluded. During risk-set matching, five LC patients were not matched to control subjects and were excluded, and another 331 patients entered into the study as control subjects of another LC patient were excluded.

Additionally, 87 LC patients were excluded due to a less than

\begin{tabular}{|c|}
\hline $\begin{array}{l}\text { Systemic stratified random sampling of } 1,000,000 \text { people } \\
\text { from a target population of } 48,222,537\end{array}$ \\
\hline Patients who did not meet incident LC inclusion criteria $(n=989,406)$ \\
\hline Patients who meet incident LC inclusion from 2002 to $2015(n=10,594)$ \\
\hline $\begin{array}{l}\text { Excluded patients }(n=4,785) \\
\text { - Patients with incident LC during the washout period of } 2002 \text { to } 2006(n=4,354) \\
\text { - Patients with incident LC did not matched to control subjects on risk-set matching }(n=5) \\
\text { - Patients with incident LC entered into the study as control subjects of another } L C \text { patients }(n=331) \\
\text { - Patients with incident LC less than 1:2 matching ratio }(n=87) \\
\text { - Patients with incident LC less than } 18 \text { years old }(n=8)\end{array}$ \\
\hline Finally enrolled incident $L C$ patients $(n=5,809)$ \\
\hline $1: 2$ matched control for incident LC patients $(n=11,618)$ \\
\hline
\end{tabular}

Figure 1. Study flow and disposition of the patients. LC, liver cirrhosis. 
1:2 matching ratio, and eight LC patients younger than 18 years were excluded. Finally, 5,809 incident LC patients and their 11,618 matched controls were analyzed in this study (Fig. 1). The mean period of follow-up was 4.20 years, generating 73,261 person years. During follow-up, a total of 61 suicide cases was identified. Table 1 shows the baseline characteristics of the matched cohorts. Mean age was 56.3 years, and $28.6 \%$ of the patients were female. The highest standardized difference in number of outpatient visits was $5.19 \%$.

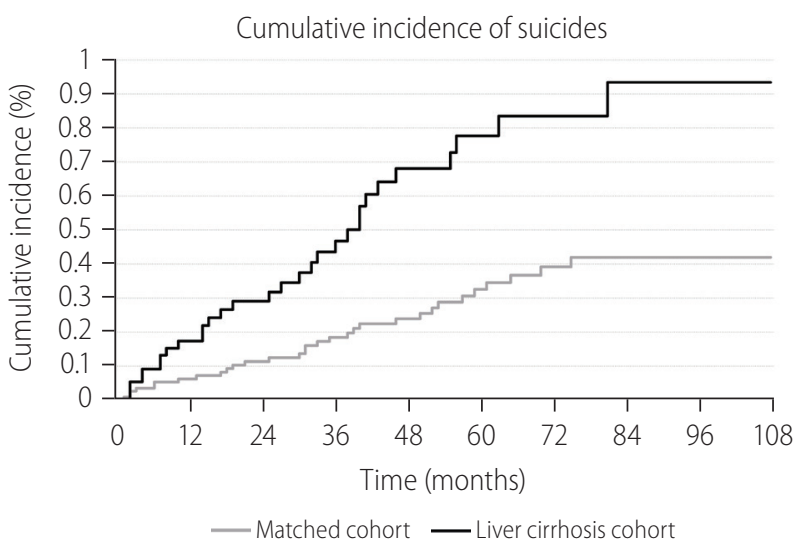

Figure 2. Comparison of cumulative incidence of suicide between incident liver cirrhosis patients and their risk set-matched controls. $P$-value for stratified log-rank test $=0.002$.

Table 2. Cumulative incidence of suicide according to survival duration after LC diagnosis

\begin{tabular}{lcc}
\hline \multirow{2}{*}{ Time frame } & \multicolumn{2}{c}{ Cumulative incidence $(\%)$ of suicide $(95 \% \mathrm{Cl})$} \\
\cline { 2 - 3 } & Liver cirrhosis cohort & Matched cohort \\
\hline 1 year & $0.17(0.09-0.32)$ & $0.06(0.03-0.13)$ \\
\hline years & $0.29(0.17-0.48)$ & $0.11(0.06-0.20)$ \\
\hline years & $0.47(0.29-0.72)$ & $0.18(0.11-0.29)$ \\
\hline years & $0.68(0.45-0.99)$ & $0.24(0.15-0.36)$ \\
\hline years & $0.78(0.52-1.13)$ & $0.33(0.22-0.47)$ \\
\hline 6 years & $0.83(0.56-1.21)$ & $0.39(0.27-0.56)$ \\
\hline years* & $0.93(0.61-1.37)$ & $0.42(0.28-0.60)$ \\
\hline years* & $0.93(0.61-1.37)$ & $0.42(0.28-0.60)$ \\
\hline years* & $0.93(0.61-1.37)$ & $0.42(0.28-0.60)$ \\
\hline
\end{tabular}

Cumulative incidence was calculated by product limit (Kaplan-Meier) method of survival probability.

LC, liver cirrhosis; $\mathrm{Cl}$, confidence interval.

*There was no suicide event between 82 and 108 months of survival time.

\section{Suicide risk in LC}

Cumulative incidence of suicide during the entire follow-up period showed a statistically significant difference between $L C$ and matched-control cohort ( $P=0.002$ for stratified log-rank test) (Fig. 2). The 5 -year cumulative incidence risk of suicide was $0.78 \%$ (95\% Cl, 0.52-1.13) for the LC cohort and 0.33\% (95\% Cl, 0.220.47) for the matched-control cohort (Table 2). During the entire follow-up period, among 5,809 LC patients 30 suicides were identified during 20,930 person-years (IR, 143.3 per 100,000 person-years; $95 \% \mathrm{Cl}, 100.2-205.1)$. The LC patients were 2.37 times more likely to commit suicide than their matched controls (HR, 2.37; 95\% Cl, 1.44-3.88). Moreover, increased suicide risk was predominantly observed within the first 2 years of follow-up (HR, 2.59; 95\% Cl, 1.20-5.60), among patients in the 18-49-year-old group (HR, 3.72; 95\% Cl, 1.45-9.56) (Fig. 3).

\section{Subgroup analysis for suicide risk according to alcohol use, LC severity, and use of anti-depressant}

In subgroup analyses, non-alcoholic LC patients had a 2.04 times higher suicide risk than the matched cohort (HR, 2.04; 95\% Cl, 1.16-3.58), and alcoholic LC patients had a 3.29 times higher suicide risk compared with the matched cohort (HR, 3.29; 95\% $\mathrm{Cl}, 1.66-6.52)$. However, when comparing the suicide risk of nonalcoholic LC patients and alcoholic LC patients, the suicide risk of alcoholic LC patients was 1.62 times higher, although there was no statistical significance $(\mathrm{HR}, 1.62 ; 95 \% \mathrm{Cl}, 0.77-3.40$; $P=0.206)$. Moreover, compensated LC (HR, 2.03; 95\% Cl, $1.18-$ 3.49) and decompensated ( $\mathrm{HR}, 4.42 ; 95 \% \mathrm{Cl}, 2.05-9.52) \mathrm{LC}$ patients were more likely to commit suicide compared to their matched controls. Interestingly, as a result of a comparative analysis of suicide risk between compensated and decompensated LC patients, the suicide risk of decompensated LC patients was 2.18 times higher, although the difference was not significant (HR, 2.18; 95\% Cl, 0.96-4.94; $P=0.062)$. Among ever-users of antidepressants, increased risk of suicide was observed in LC patients, although the association was not significant $(\mathrm{HR}, 1.97 ; 95 \% \mathrm{Cl}$, 0.55-7.13; $P=0.301)$. Among patients not taking anti-depressants, the risk of suicide in LC patients was significantly 2.44 times higher compared to that of the matched cohort (HR, 2.44; 95\% Cl, 1.42-4.17; $P=0.001$ ) (Fig. 4). Results of Fine and Gray sub-distribution hazards model were similar to those of the causespecific model. 


\section{CLINCAL and MOLECULAR}

Volume_27 Number_2 April 2021

\section{DISCUSSION}

Patients with various chronic diseases are known to exhibit a high rate of suicidal tendencies. For example, according to a national population-based study conducted in the United Kingdom, overall standardized mortality ratio (SMR) was as high as 1.20

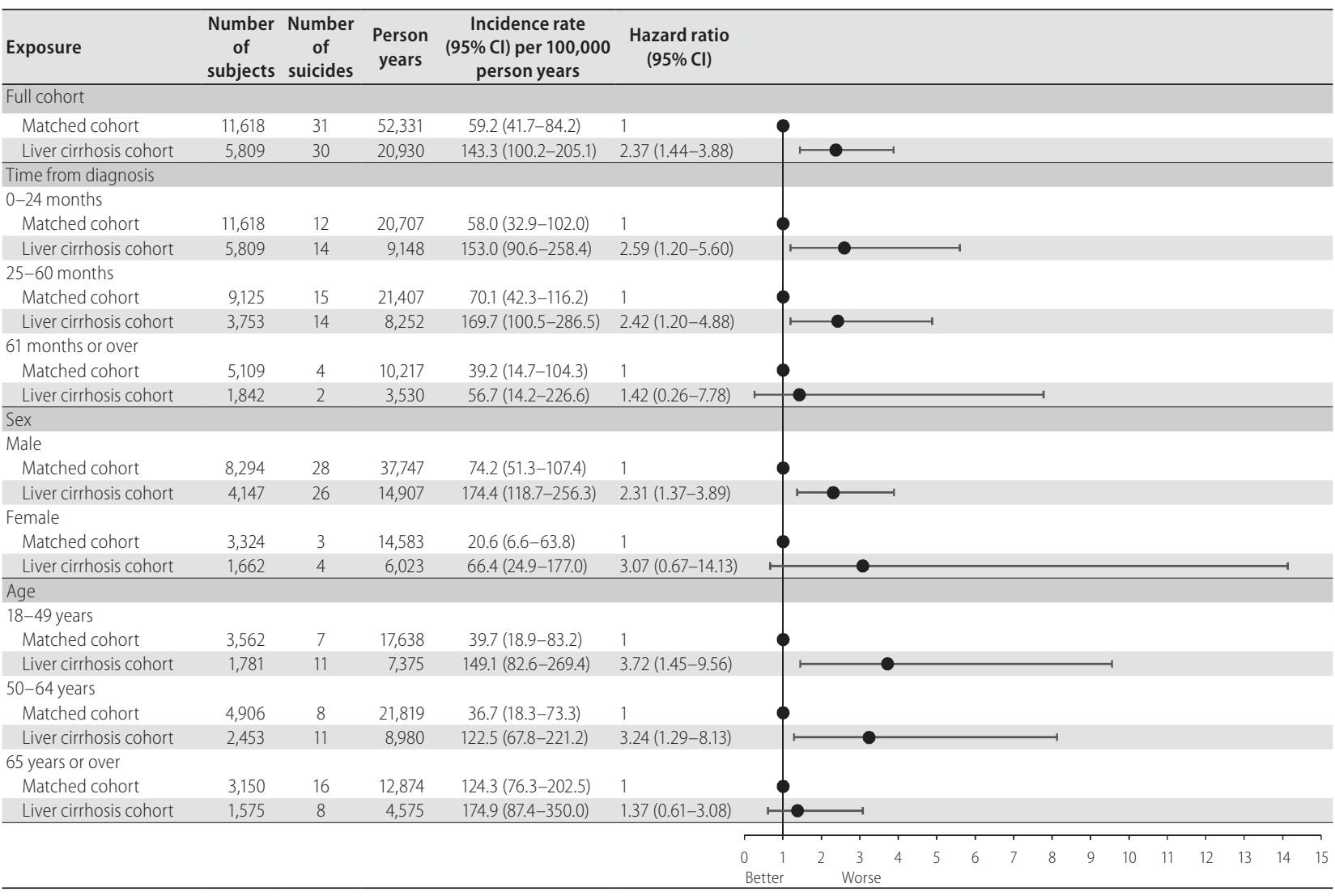

Figure 3. Comparable analysis of suicide rate for the relationship between liver cirrhosis and risk of suicide. Cl, confidence interval.

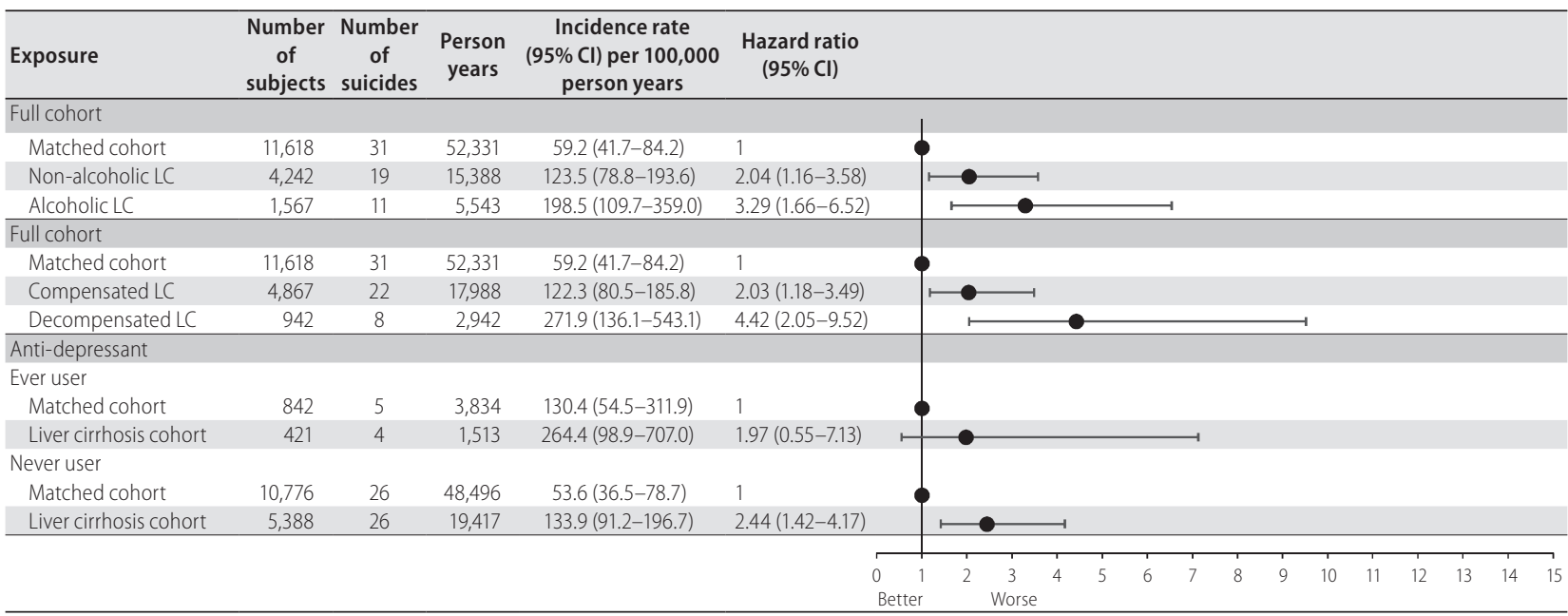

Figure 4. Subgroup analysis of suicide rate according to alcoholic etiology of LC, compensation of LC, and anti-depressant use. Cl, confidence interval; LC, liver cirrhosis. 
$(95 \% \mathrm{Cl}, 1.16-1.25)$ and was highest $(2.74 ; 95 \% \mathrm{Cl}, 2.52-2.98)$ within the first 6 months after being diagnosed with cancer. ${ }^{32}$ In addition, a retrospective, population-based study using data from the Surveillance, Epidemiology, and End Results program between 1973 and 2014, reported that the SMR in cancer patients was as high as $4.44(95 \% \mathrm{Cl}, 4.33-4.55) .{ }^{2}$ Moreover, according to a study of end-stage renal disease patients in the USA, the overall standardized incidence ratio for suicide from 1995 to 2001 was $1.84(95 \% \mathrm{Cl}, 1.50-2.27)$ and patients with end-stage renal disease had an $84 \%$ higher suicide rate than the general USA population. ${ }^{3}$ In addition, a study conducted in Taiwan found that patients with heart failure had a 1.68 times increase in suicide risk compared with age-, sex-, and residence area-matched-control subjects, with the highest risk being 7 -fold in the first 6 months after diagnosis of heart failure. ${ }^{4}$

Therefore, it is necessary to examine suicide risk in patients with chronic liver disease. Indeed, almost one in six patients with cirrhosis has moderate or higher depression than controls, and nearly $50 \%$ of these patients have moderate or higher anxiety symptoms. ${ }^{33}$ In patients with liver cancer, the overall SMR for suicide was $1.56(95 \% \mathrm{Cl}, 0.84-2.90)$, which was higher than that of the general population, although the difference was not significant. ${ }^{32}$ Previous studies have investigated the relationship between liver disease and suicide attempt. According to a survey of 43,093 adults older than 18 years in the USA, lifetime rates of suicide attempts among participants with major depression were higher in participants with liver disease (33.2\%) compared with those without liver disease (13.7\%; odds ratio [OR], 3.1; 95\% Cl, 1.3-7.6). ${ }^{5}$ These findings from a liver disease-specific study are concordant with our findings and support the idea that patients with $\mathrm{LC}$ are at higher risk of suicide. In addition, according to a nationwide population-based cohort study in South Korea, the OR for risk of suicide in patients diagnosed with alcoholic liver disease during their lifetime was $5.62(95 \% \mathrm{Cl}, 4.70-6.72)$ compared with ageand sex-matched non-suicidal living controls, and the adjusted OR was $2.39(95 \% \mathrm{Cl}, 1.88-3.04) .{ }^{34}$ In particular, among patients diagnosed with alcoholic liver disease, females had an adjusted OR of $10.68(95 \% \mathrm{Cl}, 4.04-28.21)$, which was significantly higher than adjusted OR of $1.94(95 \% \mathrm{Cl}, 1.48-2.54)$ in males. ${ }^{34}$ One possible mechanism for these findings is related to the preliminary finding that chronic liver diseases are associated with depression. ${ }^{35}$ Stigmatization of patients with cirrhosis is another possible cause. Patients with cirrhosis may experience internalized stigma due to their physical disorder, and this may be a significant risk factor for suicide. ${ }^{36}$ No study has been performed to directly in- vestigate suicide risk in patients newly diagnosed with LC.

South Korea not only exhibits one of the highest suicide rates in the world, but also the eighth highest mortality rate from chronic liver disease. Chronic liver disease accounted for $13.3 \%$ of total deaths in 2016, illustrating it as a serious disease in South Korea. ${ }^{37}$ Therefore, the Korean population provides the optimal research conditions to analyze the risk of suicide due to chronic liver disease, such as LC. Because several physical, psychological, and socioeconomic factors play a role in suicide risk, we set an adjusted matched cohort using propensity scores for patients with LC and analyzed the data based on these two populations. Our study indicates that 30 of 5,809 patients newly diagnosed with LC had a potentially preventable death during the observation period. Moreover, when we specifically analyzed the characteristics of patients who faced higher risk, one of the influencing factors was age. The 18-49-year-old age group of patients diagnosed with LC exhibited the highest risk of suicide, followed by the 50-64-year-old age group, and the 65 years and older group. This is in contrast to the notion that risk of suicide increases with age in the general population. Moreover, according to the results of our analyses, the suicide rate was high within 5 years after being diagnosed with LC, with a particularly high risk in the first 2 years. Although suicide is difficult to predict, our findings suggest that psychological support is essential for all patients diagnosed with LC, especially for those newly diagnosed. Therefore, we suggest that screening and psychological support to pre-empt suicidal tendencies in patients is necessary immediately after being diagnosed with LC.

Our study has several strengths. First, use of nationwide insurance claim data allowed a well-represented study. Because the study was conducted on the whole population and was not limited to a specific hospital or organization, the enrolled study subjects can be considered to represent the whole population in an unbiased manner. Second, the research question cannot be addressed without using a nationwide cohort based on insurance claim data, because retrospective studies or randomized controlled trials cannot conclude a relationship between suicide and LC. Suicide is an unambiguous cause of death that is unlikely to be misidentified. Third, patients enrolled in this study were tracked using insurance claim data due to the nature of the study, so there was no follow-up loss, except in rare cases of immigration or imprisonment.

There are some inherent limitations to our study. First, based on use of insurance claim data, the exact diagnosis of LC may be inaccurate. In most cases, an extensive diagnosis code was applied 
to patients' insurance coverage of medical examination, treatment modality, and drug prescription could be added in the course of patient care in hospitals or clinics. To overcome these limitations, this study used not only the two earliest diagnoses, but also patients who were admitted more than twice as outpatients and more than one for hospitalization. Second, since these study data are derived from insurance claim data, clinical information is limited. An additional limitation is that specific clinical information, such as blood test results, was not included. Third, because it is a study related to suicide in patients with $L C$, there is a possibility that confounding variables can influence the variables of cirrhosis and suicide. In addition, due to lack of information, some important variables such as severity of LC were not included in the model to calculate propensity score. To overcome these pitfalls, we selected and used a 1:2 matched cohort using a propensity score. Even so, residual confounding factors could be present after propensity score matching. Thus, we tried to overcome these shortcomings by performing Cox regression analysis considering social and economic indicators such as $\mathrm{CCl}$ score and income level. Lastly, since the diagnosis code is masked due to privacy reasons in patients with depression, classification was performed according to anti-depressant prescription to evaluate suicide risk. However, this could not accurately include all depressed patients, as those without anti-depressant description were excluded, even if they were admitted to the hospital.

In conclusion, our study found an increased risk of suicide in patients with new onset LC, especially during the early period following diagnosis and in relatively younger patients. In addition, our study results have several important clinical implications. When newly diagnosed with $L C$, patients worry about the physical manifestations of the disease and are frustrated that it cannot be cured without liver transplantation. Therefore, medical services for psychological and social support, in addition to medical services for physical problems, are essential for patients diagnosed with $L C$. These measures are needed not only in the initial stage following $L C$ diagnosis, but also for at least 2 years after diagnosis of LC. There are many ways to address the mental problems of these patients. In the study of patients with chronic liver disease, an integrated care model created by mental health providers and clinicians has been recommended in place of the usual care model. In particular, active care from experts and psychological intervention is necessary to alleviate suicide risk due to societal and self-stigmatization of physical health issues of patients with cirrhosis. Through the integrated care model, specific risk factors were identified during screening, treatment methods were sug- gested, and results were suggested to promote clinical and psychiatric improvements through regular follow-up observations. Currently, the Metropolitan Mental Health Center and the Metropolitan Suicide Prevention Center are in operation in South Korea; however, there is no specialized institution for patients with cirrhosis. Therefore, a support system including a hepatologist, psychologist, and mental health provider could result in better management of patients with cirrhosis.

\section{Authors' contributions}

Substantial contributions to conception and design, acquisition of data, or analysis and interpretation of data: S. Y. J., H. S. E, drafting the article or revising it critically for important intellectual content: W. S. R., B. S. L., S. H. K., final approval of the version to be published: S. Y. J., H. S. E. All of the authors reviewed the manuscript.

\section{Acknowledgements}

This study is based on data sourced from the Korean National Health Insurance Service (research administration number, NHIS2020-2-011), and the results of the study are not related to the National Health Insurance Service. All of the authors have declared no competing financial interests.

\section{Conflicts of Interest}

The authors have no conflicts to disclose.

\section{REFERENCES}

1. Gürhan N, Beşer NG, Polat Ü, Koç M. Suicide risk and depression in individuals with chronic illness. Community Ment Health J 2019;55:840-848.

2. Zaorsky NG, Zhang Y, Tuanquin L, Bluethmann SM, Park HS, Chinchilli VM. Suicide among cancer patients. Nat Commun 2019;10:207.

3. Kurella M, Kimmel PL, Young BS, Chertow GM. Suicide in the United States end-stage renal disease program. J Am Soc Nephrol 2005;16:774-781.

4. Liu CH, Wang JH, Weng SC, Cheng YH, Yeh MK, Bai MY, et al. Is heart failure associated with risk of suicide? J Card Fail 2018;24:795800.

5. Le Strat Y, Le Foll B, Dubertret C. Major depression and suicide attempts in patients with liver disease in the United States. Liver Int 2015;35:1910-1916.

6. Jang JW. Current status of liver diseases in Korea: liver cirrhosis. Korean J Hepatol 2009;15 Suppl 6:\$40-\$49. 
7. Lee $Y R$, Cho B, Jo MW, Ock M, Lee $D$, Lee $D$, et al. Measuring the economic burden of disease and injury in Korea, 2015. J Korean Med Sci 2019;34(Suppl 1):e80.

8. Bianchi G, Marchesini G, Nicolino F, Graziani R, Sgarbi D, Loguercio C, et al. Psychological status and depression in patients with liver cirrhosis. Dig Liver Dis 2005;37:593-600.

9. Perng CL, Shen CC, Hu LY, Yeh CM, Chen MH, Tsai CF, et al. Risk of depressive disorder following non-alcoholic cirrhosis: a nationwide population-based study. PLoS One 2014;9:e88721.

10. Seo JH, Ryu HS, Lee YY, Kim MJ, Choi YS. A study on depression, anxiety and illness symptoms according to severity classification of liver cirrhosis patients in South Korea. Biomed Res 2018;29:32433248.

11. Zhu HP, Gu YR, Zhang GL, Su YJ, Wang KE, Zheng YB, et al. Depression in patients with chronic hepatitis $B$ and cirrhosis is closely associated with the severity of liver cirrhosis. Exp Ther Med 2016;12:405-409.

12. Leutscher PD, Lagging M, Buhl MR, Pedersen C, Norkrans G, Langeland $\mathrm{N}$, et al. Evaluation of depression as a risk factor for treatment failure in chronic hepatitis C. Hepatology 2010;52:430-435.

13. Corruble E, Barry C, Varescon I, Falissard B, Castaing D, Samuel D. Depressive symptoms predict long-term mortality after liver transplantation. J Psychosom Res 2011;71:32-37.

14. Singh N, Gayowski T, Wagener MM, Marino IR. Depression in patients with cirrhosis. Impact on outcome. Dig Dis Sci 1997;42:14211427.

15. Seong SC, Kim YY, Khang YH, Heon Park J, Kang HJ, Lee $H$, et al. Data resource profile: the national health information database of the national health insurance service in South Korea. Int J Epidemiol 2017:46:799-800.

16. Lee J, Lee JS, Park SH, Shin SA, Kim K. Cohort profile: the national health insurance service-national sample cohort (NHIS-NSC), South Korea. Int J Epidemiol 2017;46:e15.

17. Lapointe-Shaw L, Georgie F, Carlone D, Cerocchi O, Chung H, Dewit $Y$, et al. Identifying cirrhosis, decompensated cirrhosis and hepatocellular carcinoma in health administrative data: a validation study. PLoS One 2018;13:e0201120.

18. Driver RJ, Balachandrakumar V, Burton A, Shearer J, Downing A, Cross $T$, et al. Validation of an algorithm using inpatient electronic health records to determine the presence and severity of cirrhosis in patients with hepatocellular carcinoma in England: an observational study. BMJ Open 2019;9:e028571.

19. Lu M, Chacra W, Rabin D, Rupp LB, Trudeau S, Li J, et al. Validity of an automated algorithm using diagnosis and procedure codes to identify decompensated cirrhosis using electronic health records. Clin Epidemiol 2017;9:369-376.

20. Kwon JW, Tchoe HJ, Lee J, Suh JK, Lee JH, Shin S. The impact of national surveillance for liver cancer: results from real-world setting in
Korea. Gut Liver 2020;14:108-116.

21. Suissa S. Immortal time bias in pharmaco-epidemiology. Am J Epidemiol 2008;167:492-499.

22. Kim YI, Kim YY, Yoon JL, Won CW, Ha S, Cho KD, et al. Cohort profile: National Health Insurance Service-senior (NHIS-senior) cohort in Korea. BMJ Open 2019;9:e024344.

23. Li YP, Propert KJ, Rosenbaum PR. Balanced risk set matching. J Am Stat Assoc 2001;96:870-882.

24. Lu B. Propensity score matching with time-dependent covariates. Biometrics 2005;61:721-728.

25. Quan H, Sundararajan V, Halfon P, Fong A, Burnand B, Luthi JC, et al. Coding algorithms for defining comorbidities in ICD-9-CM and ICD-10 administrative data. Med Care 2005;43:1130-1139.

26. Andersen LW, Granfeldt A, Callaway CW, Bradley SM, Soar J, Nolan $J P$, et al. Association between tracheal intubation during adult inhospital cardiac arrest and survival. JAMA 2017;317:494-506.

27. Suissa S, Moodie EE, Dell'Aniello S. Prevalent new-user cohort designs for comparative drug effect studies by time-conditional propensity scores. Pharmacoepidemiol Drug Saf 2017;26:459-468.

28. Yoo KD, Kim CT, Kim MH, Noh J, Kim G, Kim H, et al. Superior outcomes of kidney transplantation compared with dialysis: an optimal matched analysis of a national population-based cohort study between 2005 and 2008 in Korea. Medicine (Baltimore) 2016;95:e4352.

29. Austin PC. An introduction to propensity score methods for reducing the effects of confounding in observational studies. Multivariate Behav Res 2011;46:399-424.

30. Rasouliyan L, Plana E, Aguado J. Considerations in the use of propensity scores in observational studies. RTI Health Solutions web site, <https://www.rtihs.org/sites/default/files/28177\%20Rasouliyan $\% 202016 \% 20$ Considerations $\% 20$ in $\% 20$ the $\% 20$ use $\% 20$ of $\% 20$ propensity \%20scores\%20in\%20observational\%20studies.pdf>. Accessed 10 Aug 2020.

31. Austin PC. The use of propensity score methods with survival or time-to-event outcomes: reporting measures of effect similar to those used in randomized experiments. Stat Med 2014;33:1242 1258.

32. Henson KE, Brock R, Charnock J, Wickramasinghe B, Will O, Pitman A. Risk of suicide after cancer diagnosis in England. JAMA Psychiatry 2019;76:51-60.

33. Hernaez R, Kramer JR, Khan A, Phillips J, McCallister K, Chaffin K, et al. Depression and anxiety are common among patients with cirrhosis. Clin Gastroenterol Hepatol. 2020 Aug 21. doi: 10.1016/ j.cgh.2020.08.045.

34. Na EJ, Lee H, Myung W, Fava M, Mischoulon D, Paik JW, et al. Risks of completed suicide of community individuals with ICD-10 disorders across age groups: a nationwide population-based nested casecontrol study in South Korea. Psychiatry Investig 2019;16:314-324. 


\section{CLINCAL and MOLECULAR}

Volume_27 Number_2 April 2021

35. Lee K, Otgonsuren M, Younoszai Z, Mir HM, Younossi ZM. Association of chronic liver disease with depression: a population-based study. Psychosomatics 2013;54:52-59.

36. Carpiniello B, Pinna F. The reciprocal relationship between suicidal- ity and stigma. Front Psychiatry 2017;8:35.

37. Kim HJ, Chu H, Lee S. Factors influencing on health-related quality of life in South Korean with chronic liver disease. Health Qual Life Outcomes 2018;16:142. 